\title{
Estudio del parasitismo gastrointestinal y externo en caiquén Chloephaga picta Gmelin, 1789 (Aves, Anatidae) en la región de Magallanes, Chile
}

\author{
DANIEL GONZÁLEZ*, OSCAR SKEWES*, CORITA CANDIA*, \\ RICARDO PALMA** y LUCILA MORENO*
}

\author{
STUDY OF INTESTINAL ENDOPARASITES AND ECTOPARASITES IN CAIQUÉN \\ Chloephaga picta Gmelin, 1789 (BIRDS, ANATIDAE) IN \\ THE REGION OF MAGALLANES, CHILE
}

The parasites of 16 Magellan geese (Chloephaga picta picta: 8 females and 8 males), captured in May 2002 in the Commune of Río Verde, Province of Magallanes, XII Región, Chile, were studied. All the birds from this study were found positive for the endoparasitic nematode Heterakis dispar. In one of the birds, proglotides were found which, from a morphological analysis, belong to the family Hymenolepidae. The total number of specimens of $\boldsymbol{H}$. dispar was greater in male geese than in females. The female/male ratio was 1.22. The larva/adult ratio was 0.41. No parasites were found in a study of goose faeces. An $87.5 \%$ of the birds were parasitised by one or more of the following louse species: Holomenopon brevithoracicum, Anaticola marginella, Anatoecus dentatus and Anatoecus icterodes, all of them belonging to the Order Phthiraptera. All these species represent new louse records from Magellan geese for Chile, as well as a new host-louse record for A. dentatus on C. picta.

Key words: Aves, Chloephaga picta, Heterakis dispar, Phthiraptera, endoparasites, ectoparasites, new records.

\section{INTRODUCCIÓN}

El caiquén (Chloephaga picta (Gmelin, 1789)) es el más abundante de las cinco especies de gansos sudamericanos ${ }^{1}$. En Chile, la subespecie es $C$. picta picta, la cual se distribuye desde Colchagua hasta el cabo de Hornos ${ }^{2}$. Es un ave que se concentra en ambientes de estepa y matorral especialmente en áreas de vegas y pastos blandos $^{3}$. C. picta es estrictamente vegetariana, se alimenta de pastos blandos y semillas ${ }^{4,5}$. Es gregaria, llegando a formar bandadas que alcanzan el centenar de individuos ${ }^{4}$.

Hasta la fecha, solamente existe un estudio de los endoparásitos de C. picta leucoptera ${ }^{6}$ en las Islas Malvinas (= Falkland Islands), el cual describió parásitos céstodos himenolépidos en el intestino delgado, nemátodos pertenecientes a la especie Amidostomum anseris en la molleja, y las especies Heterakis dispar y Trichostrongylus

Facultad de Ciencias Veterinarias, Departamento de Ciencias Pecuarias, Universidad de Concepción, Chillán, Chile.

** Museum of New Zealand Te Papa Tongarewa, P.O. Box 467, Wellington, New Zealand danigonz@udec.cl 
tenuis en el ciego.

En relación a los ectoparásitos, se han descrito la especies de piojo Anaticola marginella (Insecta, Phthiraptera, Ischnocera) ${ }^{7}$ y Holomenopon brevithoracicum (Insecta, Phthiraptera, Amblycera $)^{8}$ sobre $C$. picta leucoptera, en ambos casos para las Islas Malvinas. También se han reportado en Argentina, $H$. brevithoracicum sobre el hospedador tipo, Cygnus melanocoryphus ${ }^{9}$ y tres especies de Phthiraptera sobre $C$. picta picta: $H$. brevithoracicum, A. marginella (como A. marginellus) y una subespecie indeterminada de Anatoecus icterodes, las dos últimas pertenecientes al suborden Ischnocera ${ }^{10}$. No se han reportado antecedentes de la presencia de ácaros ni dípteros parásitos en la especie $C$. picta.

\section{MATERIAL Y MÉTODOS}

Entre el 7 y el 9 de abril del año 2002 se obtuvieron durante la temporada de caza (Ley $\mathrm{N}^{\mathrm{o}}$ 19.473) 16 caiquenes de Magallanes (C. picta picta) en la Comuna de Río Verde 52 $34^{\circ} 07^{\prime}$ 'S, 71'32’44” W, Provincia de Magallanes, XII Región. Los caiquenes fueron cazados como parte del proyecto "Estudio de las relaciones forrajeras de los principales herbívoros silvestres con la ganadería de la XII Región, Chile".

Posterior a la caza, cada ejemplar fue pesado, medido e identificado su sexo. Luego fueron almacenados en forma individual en bolsas plásticas herméticas para posteriormente ser procesados en el laboratorio y así no perder ectoparásitos.

Los ectoparásitos fueron colectados en forma manual desde cada ejemplar el mismo día de su captura, se conservaron en alcohol $70 \%$ y posteriormente fueron limpiados en $\mathrm{KOH}$ al $20 \%$, llevados por soluciones ascendentes de alcohol ( $40 \%, 80 \%$ y $100 \%)$, aclarados durante 24 horas en aceite de clavo, para finalmente ser montados en Bálsamo de Canadá ${ }^{11}$. Las técnicas de colecta, fijación y tinción de los endoparásitos se basó en una metodología preestablecida ${ }^{12}$. De las porciones de recto de cada ejemplar se obtuvo una muestra de material fecal, la cual fue analizada mediante por el método de flotación tradicional ${ }^{13}$.

A partir de los endoparásitos aislados se calculó la intensidad media, el rango y la abundancia, cálculos que se realizaron en base a lo descrito por otros investigadores ${ }^{14,15}$. Se realizó un análisis estadístico a fin de determinar si existe relación entre el sexo de las aves capturadas y el nivel de infección de parásitos gastrointestinales encontrados. Además, se buscó determinar si existían más endoparásitos hembras o machos en los caiquenes capturados. Para ambos estudios se utilizó la prueba estadística de $t$ de Student.

\section{RESULTADOS Y DISCUSIÓN}

Los 16 caiquenes analizados se encontraron parasitados por algún nematodo y la única especie que se encontró fue $H$. dispar. Esta especie es de distribución cosmopolita y se ha reportado en aves de los géneros Anser, Anas, Branta, Tadorna, Strix, Surnia, Glaucidium, Alectoris, Cairina, Numida, Bernicla, Cygnopsis y Chloephaga ${ }^{16}$ e incluso en gansos de nieve adultos (Chen caerulescens caerulescens) de Manitoba, Canadá ${ }^{17}$.

De acuerdo a lo expuesto en la Tabla 1, en los caiquenes fueron aislados 8.622 ejemplares de la especie $H$. dispar, de los cuales 3.193 eran machos, 4.471 eran hembras y 958 larvas. La relación hembra/macho fue en promedio de 1,22 hembra por cada macho y la relación larva/ adulto fue en promedio 0,41 larvas por cada adulto.

Siguiendo las pautas descritas ${ }^{14,15}$, el presente estudio arrojó para $H$. dispar una abundancia de $100 \%$. La intensidad media fue de 199,56 para los machos, 279,44 para las hembras y 59,88 para las larvas. El rango correspondió a 3-1.263 endoparásitos.

Se aislaron una mayor cantidad de parásitos hembras que machos, diferencia significativa con $\alpha=0,003 \%$, lo cual se debe a que son éstas las encargadas de la ovoposición, por lo que se asume que su periodo de vida es más prolongado.

Como lo citan diversos autores ${ }^{18-20}$, las especies del género Heterakis son parásitas principalmente del ciego, dato que coincide con los parásitos aislados en el ciego en el presente estudio, aunque también es posible aislarlo en ciertas ocasiones en el intestino delgado de las aves $^{21}$. Los individuos que se aislan en distintas partes de intestino delgado y grueso se podría deber a la migración de los vermes post mortem del hospedador ${ }^{22}$.

Los caiquenes machos estaban significativamente más infectados que las hembras $(\alpha$ $=0,04 \%)$, lo cual coincide con otro autor quien confirma esta tendencia (tanto en mamíferos y 
Parasitismo en caiquén Chloephaga picta (Anatidae) en Magallanes Chile - D. González et al.

Tabla 1. Cantidad de nematodos parásitos (Heterakis dispar) aislados de 16 Caiquenes cazados en la Provincia de Magallanes en mayo del año 2002

\begin{tabular}{|c|c|c|c|c|c|c|}
\hline $\begin{array}{l}\text { Caiquén } \\
\mathbf{N}^{\circ}\end{array}$ & $\begin{array}{c}\text { Machos } \\
(\%)\end{array}$ & $\begin{array}{c}\text { Hembras } \\
(\%)\end{array}$ & $\begin{array}{c}\text { Larvas } \\
(\%)\end{array}$ & Totales & $\begin{array}{c}\text { Relación } \\
\text { Larva/ } \\
\text { adultos }\end{array}$ & $\begin{array}{c}\text { Relación } \\
\text { Hembra/ } \\
\text { macho }\end{array}$ \\
\hline 1 & $349(37.05)$ & $547(58.07)$ & $46(4.88)$ & 942 & $0.05 / 1$ & $1.57 / 1$ \\
\hline 2 & $80(37.92)$ & $93(38.11)$ & $71(29.10)$ & 244 & $0.41 / 1$ & $1.16 / 1$ \\
\hline 3 & $24(10.17)$ & $52(22.03)$ & $160(67.80)$ & 236 & $0.68 / 1$ & $2.16 / 1$ \\
\hline 4 & $93(24.67)$ & $177(46.95)$ & $107(28.38)$ & 377 & $0.40 / 1$ & $1.9 / 1$ \\
\hline 5 & $347(31.46)$ & $599(54.31)$ & $157(14.23)$ & 1103 & $0.17 / 1$ & $1.73 / 1$ \\
\hline 6 & $13(46.43)$ & $6(21.43)$ & $9(14.32)$ & 28 & $0.47 / 1$ & $0.46 / 1$ \\
\hline 7 & $342(39.6)$ & $508(58.80)$ & $14(1.62)$ & 864 & $0.02 / 1$ & $1.49 / 1$ \\
\hline 8 & $1(33.33)$ & $0(0.00)$ & $2(66.67)$ & 3 & $2.00 / 1$ & $0.00 / 1$ \\
\hline 9 & $379(43.22)$ & $469(53.48)$ & $29(3.30)$ & 877 & $0.03 / 1$ & $1.24 / 1$ \\
\hline 10 & $101(36.07)$ & $170(60.71)$ & $9(3.21)$ & 280 & $0.03 / 1$ & $1.68 / 1$ \\
\hline 11 & $48(23.30)$ & $43(20.87)$ & $115(55.83)$ & 206 & $1.26 / 1$ & $0.89 / 1$ \\
\hline 12 & $334(40.93)$ & $451(55.27)$ & $31(3.80)$ & 816 & $0.04 / 1$ & $1.35 / 1$ \\
\hline 13 & $0(0.00)$ & $0(0.00)$ & $6(100)$ & 6 & $0.00 / 1$ & $0.00 / 1$ \\
\hline 14 & $415(55.63)$ & $300(40.21)$ & $31(4.16)$ & 746 & $0.04 / 1$ & $0.72 / 1$ \\
\hline 15 & $228(36.13)$ & $323(51.19)$ & $80(12.68)$ & 631 & $0.15 / 1$ & $1.42 / 1$ \\
\hline 16 & $439(34.76)$ & $733(58.04)$ & $91(17.20)$ & 1263 & $0.08 / 1$ & $1.67 / 1$ \\
\hline Totales & 3193 & 4471 & 958 & 8622 & & \\
\hline Promedios & 167.3 & 253.5 & 63.3 & 484.3 & $0.41 / 1$ & $1.22 / 1$ \\
\hline
\end{tabular}

aves) $)^{23}$. Esto puede deberse a factores morfológicos como el tamaño relativo del hospedador, factores fisiológicos como diferencias en los niveles hormonales debidas a stress, inmunosupresión o a condiciones reproductivas y etológicas como comportamiento, interacciones sociales y alimentación ${ }^{24}$.

En un caiquén fueron aisladas proglótidas en el intestino delgado, las cuales morfológicamente correspondían a la familia Hymenolepidae, sin embargo, no pudimos definir la especie tal como sucedió en las Malvinas ${ }^{25}$.

Catorce de los 16 caiquenes estudiados $(87,5 \%)$ presentaron ectoparásitos, siendo la totalidad de los ejemplares aislados, sólo representantes del Orden Phthiraptera. Se identificaron las siguientes especies: $H$. brevithoracicum del suborden Amblycera, y Anatoecus dentatus, A. icterodes y Anaticola marginella del suborden Ischnocera. La especies $H$. brevithoracicum y A. marginella aisladas en el presente estudio corresponden a primeros registros de dichas especies para Chile. A. dentatus y A. icterodes son especies cosmopolitas registradas sobre más de 65 especies de aves de la familia Anatidae ${ }^{26}$. Dichos autores no incluyen al caiquén $C$. picta en sus largas listas de hospedadores conocidos para A. dentatus y $A$. icterodes. Sin embargo, se menciona una subespecie indeterminada de A. icterodes para C. picta picta en la Argentina ${ }^{10}$. Por lo tanto, el aislamiento de estas dos especies de Anatoecus en el presente estudio, representa los primeros registros sobre $C$. picta picta para Chile y, en el caso de $A$. dentatus, para el mismo hospedador en toda su distribución geográfica.

Parásitos comunes en hospedadores distintos, como las especies A. dentatus y A. icterodes, por lo general confieren a sus hospedadores estrechas relaciones de parentesco. Sin embargo, esa regla no siempre se comporta como universal, debido a que los parásitos cambian a nuevos hospedadores con más frecuencia de lo que se cree $^{27}$.

\section{RESUMEN}

Se analizaron los parásitos de 16 Caiquenes de Magallanes (Chloephaga picta picta: 8 hembras y 8 machos) cazados en el mes de mayo del año 2002 en la Comuna de Río Verde, Provincia de Magallanes, XII Región, Chile. Todas las aves estudiadas resultaron positivas para la especie endoparásita Heterakis dispar (Nematoda). En un caiquén se aislaron proglótidas que morfológicamente corresponden a la Familia 
Hymenolepidae. La carga parasitaria de $H$. dispar fue mayor en Caiquenes machos que hembras. $\mathrm{Al}$ analizar la relación hembra/macho de $H$. dispar se encontró que por cada macho aislado hubo 1,22 hembras. La relación larva/adulto fue en promedio 0,41 larvas por cada adulto. En el examen coprológico no se identificaron formas parasitarias.

En el $87,5 \%$ de las aves analizadas se aislaron alguno de los siguientes insectos ectoparásitos: Holomenopon brevithoracicum, Anaticola marginella, Anatoecus dentatus y Anatoecus icterodes, todos pertenecientes al Orden Phthiraptera. Todas estas especies aisladas representan nuevos reportes para Chile, mientras que $A$. dentatus es un nuevo registro para $C$. picta picta en toda su distribución geográfica.

\section{REFERENCIAS}

1.- DEL HOYO J, ELLIOT A, SARGATAL J. Handbook of the birds of the world. Lynx Editions, Barcelona, España. 1992.

2.- ARAYA M B, MILLIE G H. Guía de campo de las aves de Chile. Editorial Universitaria, Santiago, Chile. 2000.

3.- MARTÍNEZ D, GONZÁLEZ G. Las aves de Chile, Nueva guía de campo. Ediciones del Naturalista, Santiago, 2005. 620 p.

4.- DE LA PEÑA M. Guía de aves Argentinas. Tomo I. Ediciones L.O.L.A. (Literature of Latin America). Buenos Aires, Argentina. 1992.

5.- VENEGAS C. Aves de Magallanes. Ediciones Universidad de Magallanes, Pta Arenas,Chile. 1994.

6.- HARRADINE J. Some mortality patterns of Greater Magellan Geese on the Falkland Islands. Wildfowl. 1982; 33: 7-11.

7.- PIAGET E. Les Pédiculines. Essai Monographique. Suppl. Leide: E.J. Brill xvi 1885; 200: 1-17.

8.- PRICE R D. A review of the genus Holomenopon (Mallophaga: Menoponidae) from the Anseriformes. Ann Entomol Soc America 1971; 64: 633-46.

9.- CASTRO D DEL C, CICCHINO A C. Algunas menoponidae (Insecta- Phthiraptera) de aves bonaerenses. Rev Asoc Cienc Nat Litoral 1996; 27: 137-40.

10.- CICCHINO A C, CASTRO D DEL C. Amblycera. pp: 84-103; Ischnocera pp: 104-137. En J.J. Morrone y S. Coscarón (Ed.): Biodiversidad de artrópodos argentinos - Una perspectiva biotaxonómica. Ediciones Sur. La Plata, Argentina. 1998.

11.- PALMA R L. Slide-mounting of lice: a detailed description of the Canada balsam technique. New Zealand Entomol 1978; 6: 432-6.
12.- KINSELLA J M, FORRESTER D J. Helminth parasites of the Florida duck, Anas platyrhynchos fulvipula. Proc Helminthol Soc Wash 1972; 39: 173-6.

13.- BOCH J, SUPPERER R. Veterinärmedizinische Parasitologie. Verlag Paul Parey. Hamburg, Germany. 1992.

14.- MARGOLLS L, ESCH G W, HOLMES J C, et al. The use of ecological terms in parasitology (Report of an ad-hoc committee of Amer Soc Parasitologists) J Parasitol 1982; 68: 131-3.

15.- BUSH A O, LAFFERTY K D, LOTZ J M, SHOSTAK A W. Parasitology meets ecology on its own terms: Margolis et al., revisited. J Parasitol 1997; 83: 57583.

16.- YAMAGUTI S. The nematodes of vertebrates. Vol.III. Interscience Publisher. New York, U.S.A. 1961.

17.- CLINCHY M, BARKER I. Dynamics of parasitic infections at four sites within Lesser Snow Geese (Chen caerulescens caerulescens) from the Breeding Colony at La Pérouse Bay, Manitoba, Canada. J Parasitol 1994; 80: 663-6.

18.- LEVINE N. Tratado de parasitología veterinaria. Acribia. Zaragoza, España. 1978.

19.- SOULSBY E J L. Parasitología y enfermedades parasitarias en los animales domésticos $\left(7^{\mathrm{a}} \mathrm{ed}\right)$. Nueva Editorial Interamericana. México. 1987.

20.- VICENTE J J, RODRÍGUEZ H DE O, GOMES D C, PINTO R M. Nematoídes do Brazil. Parte IV: Nematoídes de aves. Rev Bras Zool 1995; 12 (Supl 1): 1-273.

21.- DAVIS J, ANDERSON R, KARSTAD L, TRAINER D. Enfermedades infecciosas y parasitarias de las aves silvestres. Acribia. Zaragoza, España. 1977.

22.- WEISS E. Órganos digestivos. pp.121-182. En: E. Dahme y E. Weiss (Ed.): Anatomía patológica especial veterinaria. Acribia. Zaragoza, España. 1989.

23.- POULIN R. Sexual inequalities in helminth infections: a cost of being a male?. Amer Natur 1996; 147: 237 95.

24.- ZUK M, MCLEAN K. Sex differences in parasite infections: Patterns and Processes. Int $\mathrm{J}$ Parasitol 1996; 26: 1009-24.

25.- SUMMERS R, MCADAM J. A study of interaction between geese, sheep and man in the Falkland Islands. Published by Bluntisham Books. Huntingdon, UK. 1993.

26.- PRICE R D, HELLENTAL R A, PALMA R L, et al. The Chewing lice -World checklist and biological overview. Illinois Natural History Survey. U.S.A. 2003.

27.- NOTARIO A, MARTÍN M P, BARAGAÑO J R, CASTRESANA L. Contribución al conocimiento de los insectos ectoparásitos de aves acuáticas. Bol San Veg Plagas 1994; 20: 551-9.

Agradecimientos: Agradecemos al Servicio Agrícola Ganadero de la XII Región de Chile (SAG) por el financiamiento del proyecto que permitió este estudio. 